TRABAJOS DE PREHISTORIA

68, N. ${ }^{\circ}$ 1, enero-junio 2011, pp. 175-186, ISSN: 0082-5638

doi: $10.3989 /$ tp.2011.11065

\title{
El Complexo Arqueológico dos Perdigões (Reguengos de Monsaraz, Portugal). Prospecciones Geofísicas - Campañas 2008-09
}

\author{
The Archaeological Complex at Perdigões (Reguengos de Monsaraz, Portugal). \\ The Geophysical Surveys in - 2008-09
}

\author{
José Enrique Márquez Romero (*) \\ António Carlos Valera (**) \\ Helmut Becker $(* * *)$ \\ Víctor Jiménez Jáimez (*) \\ José Suárez Padilla $(*)$
}

\section{RESUMEN}

En 1997, bajo la coordinación de Era-Arqueología, se efectuaron fotografías aéreas, prospecciones superficiales y sondeos arqueológicos en el yacimiento neolítico-calcolítico de Perdigões (Reguengos de Monsaraz, Portugal), donde, durante más de diez años, se han venido articulando las distintas actuaciones hasta disponer de un esbozo bastante aproximado de su planta. En 2006, se abrió un programa de colaboración entre diversas instituciones internacionales públicas y privadas para impulsar la investigación en Perdigões, en el seno del cual se integra un equipo de la Universidad de Málaga. Como primera aportación concreta de la cooperación hispano-lusa, las prospecciones geofísicas llevadas a cabo en 2008 y 2009 han acrecentado el conocimiento de la traza del sitio. Muestra Perdigões como la yuxtaposición de múltiples recintos de foso subcirculares, que se articulan con un área de necrópolis y un crómlech.

\begin{abstract}
In 1997, under the coordination of Era-Arqueologia, aerial photographs, surface surveys and archaeological
\end{abstract}

(*) Área de Prehistoria, Facultad de Filosofía y Letras, Universidad de Málaga, Campus de Teatinos s/n. 29071 Málaga España. V. J. J. y J. S. P. Investigadores del proyecto P08-HUM04212. Correos e.: jemarquez@uma.es; vjjaimez@gmail.com; psuarezarqueo@gmail.com

(**) Direcção do Núcleo de Investigação Arqueológica NIA, Era-Arqueología S.A. Cç. de Santa Catarina 9C. 1495-705 Cruz Quebrada - Dafundo, Oeiras. Portugal. Correo e.: antoniovalera@era-arqueologia.pt

$(* * *)$ Becker Archaeological Prospection. Loisachweg 32. D-82547 Beuerberg, Eurasburg. Alemania. Correo e.:

becker.mag@googlemail.com

Recibido: 19-II-2010; aceptado: 19-V-2010. excavations were carried out at the NeolithicChalcolithic site at Perdigões (Reguengos de Monsaraz, Portugal). As a result, a quite accurate idea of the general layout of the site was achieved, allowing archaeologists to continue working there for more than ten years. In 2006, a new collaboration project was started to boost research at Perdigones, with the participation of several international public and private institutions, amongst which is the University of Málaga. The earliest contribution of the Spanish-Portuguese cooperation is the geophysical surveys performed in 2008 and 2009, which show Perdigões as a juxtaposition of several subcircular ditched enclosures, articulated with a funerary area and a cromlech.

Palabras clave: Prehistoria Reciente; Neolítico; Calcolítico; Recintos de fosos; Megalitismo; Península Ibérica; Portugal; Alentejo; Reguengos de Monsaraz; Perdigões; Arqueometría; Prospección geofísica; Georadar; Magnetometría.

Key words: Late Prehistory; Neolithic; Chalcolithic; Ditched enclosures; Megalithism; Iberian Peninsula; Portugal; Alentejo; Reguengos de Monsaraz; Perdigões; Archaeometry; Geophysical survey; Georadar; Magnetometry.

\section{INTRODUCCIÓN}

Conocemos por Complexo Arqueológico dos Perdigões un conjunto de recintos de fosos (y posibles empalizadas) genéricamente concéntricos, un espacio de necrópolis encuadrado por el foso exterior, y un crómlech en la periferia oriental de 
los recintos, que ocupan cerca de 16 ha. Se localiza en el Concelho de Reguengos de Monsaraz, a unos $2 \mathrm{~km}$ al noroeste del núcleo urbano, en el distrito de Évora, en el Alentejo portugués (Fig. 1). Coincide, asimismo, con el límite occidental de una gran concentración megalítica, conocida desde hace bastante tiempo en la región, que cuenta con casi un centenar y medio de construcciones de esta naturaleza (menhires, antas, crómlechs...) (Leisner y Leisner 1951; Gonçalves 1999).

El complejo arqueológico se abre al valle de la Ribeira do Álamo, a partir de su desarrollo en sentido aproximado O-E, antes de su desembocadura en el gran curso fluvial de la región, el Guadiana. En dicho valle, bastante llano y de sustrato geológico granítico, predominan los suelos mediterráneos, a veces de barros, con buena fertilidad y escasa erosión. Ello contrasta con su entorno, especialmente al norte y al sur, donde lo habitual es un relieve más ondulado y sinuoso, sometido a mayores procesos erosivos, y donde la base casi siempre es pizarrosa.

Perdigões se implanta en la alargada vertiente derecha del valle de la Ribeira do Alamo, donde se incurva su curso desde el $\mathrm{N}$ hacia el E. La topografía del lugar donde se asienta el yacimiento recuerda la de un teatro griego. Por un lado, una pendiente discurre desde la base (226 m.s.n.m.), al $\mathrm{E}$, hasta un punto muy próximo a la cima amesetada de la vertiente, aunque no coincidente con ella (252 m.s.n.m.), al O. Por el otro, una pendiente, menos notable que la anterior, se extiende desde los lados septentrional y meridional hacia

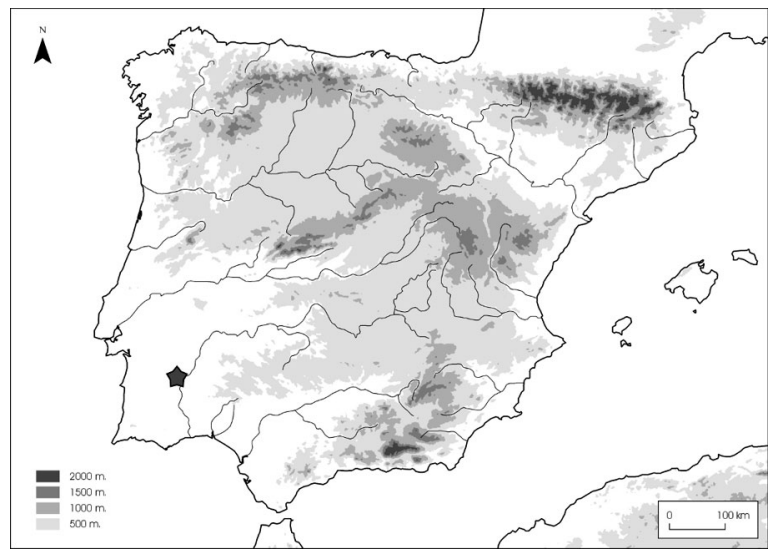

Fig. 1. Situación de Perdigões y del área de Reguengos de Monsaraz (Portugal) en la Península Ibérica. el centro del sitio. El resultado es un sitio arqueológico con visibilidad, desde su centro, limitada hacia el N, el S y el O, y una amplia visibilidad hacia el E, es decir, hacia el valle, que se abre fértil y húmedo hasta las inmediaciones de la localidad de Monsaraz. Tras ella, el Guadiana, a una quincena de kilómetros del yacimiento, a lo largo del cual se ubica la gran concentración de monumentos megalíticos de Reguengos.

\section{PRIMEROS ESTUDIOS ARQUEOLÓGICOS EN PERDIGÕES (1983-2006)}

La investigación arqueológica en el sitio comenzó de la mano de Mário Varela Gomes, cuando sólo era conocida la agrupación de menhires o cromeleque que se halla en la periferia oriental del conjunto (Gomes 1994). En el transcurso de una excavación en el mismo, en 1983, Francisco Serpa, colaborador en dichos trabajos, identificó y excavó, en un olivar cercano, lo que parecían ser algunas estructuras en negativo. A esas alturas aún no se conocía la verdadera naturaleza y dimensión del sitio arqueológico.

En 1996, la empresa propietaria de los terrenos donde se asienta la mayor parte del yacimiento, la Herdade dos Perdigões, retiró el olivar con la intención de plantar una viña. Las remociones de tierra consiguientes sacaron a la luz incontables materiales arqueológicos y posibles estructuras en negativo. Por su importancia, el yacimiento fue puesto de inmediato bajo la tutela del Instituto Portugués del Patrimonio Arquitectónico (IPPAR), el cual propició trabajos arqueológicos de diagnóstico. El promotor contrató a Era-Arqueología, entidad que, desde entonces, ha coordinado y desarrollado la investigación hasta el presente (Lago et al. 1998a, Lago et al. 1998b; Valera et al. 2007). La propia institución del IPPAR encargó, en agosto de 1997, las primeras fotografías aéreas del sitio. Ese mismo año, Era-Arqueología volvió a realizar fotos aéreas, llevadas a cabo por Manuel Ribeiro (Fig. 2).

Además de los beneficios patrimoniales, de cara sobre todo a la protección del yacimiento, desde el punto de vista científico las vistas aéreas generaron la primera imagen de conjunto del lugar. En ellas se advertía, con sorprendente claridad, la extensa superficie del sitio (16 ha) y su composición, con varios fosos claramente per- 


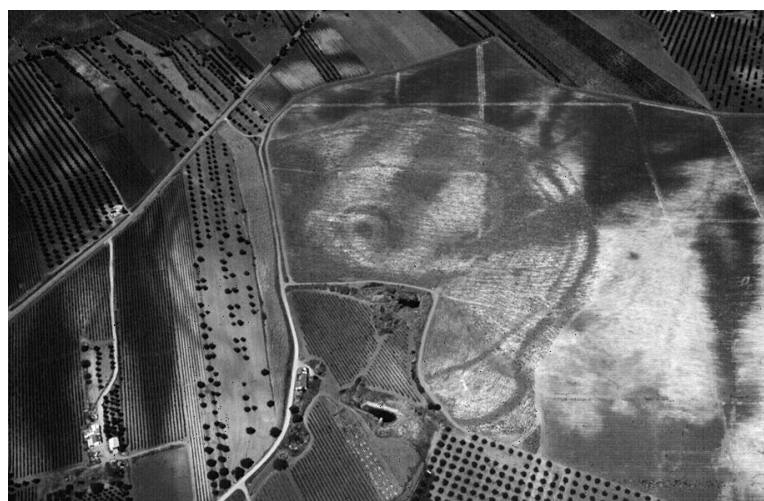

Fig. 2. Perdigões (Reguengos de Monsaraz, Portugal). Fotografía aérea del yacimiento, tomada por Manuel Ribeiro en agosto de 1997 (Lago et al. 1998a: fig. 30).

ceptibles. Los dos más exteriores, discurrían de forma prácticamente paralela hasta un punto, al $\mathrm{E}$ del sitio, en el que el foso más externo se distanciaba de su foso hermano, delimitando un sector semicircular donde se ubicaba la necrópolis. En el trazado de dichas zanjas se advertían dos discontinuidades, interpretadas, respectivamente, como las puertas noreste y sureste del recinto (Valera 2003: 157). Por otra parte, en el interior del espacio circundado se apreciaba también una gran "mancha" central, además de otros fosos de trazado más irregular, de apariencia concéntrica.

Por aquellas fechas, se emprendieron, paralelamente, labores de prospección y documentación de los restos arqueológicos perceptibles en superficie, permitiendo constatar in situ muchas de las evidencias que se dibujaban en las fotos aéreas, así como datar el sitio, de forma preliminar, en la Edad del Cobre (1). Además, al amparo de esta información, se eligieron cinco puntos en los que, en el mismo año 1997, se efectuaron sondeos destinados a determinar los daños producidos por los trabajos agrícolas y a evaluar las potencialidades arqueológicas del sitio (2) (Lago et al. 1998a,

(1) Hoy sabemos que la actividad humana en el lugar se remonta al menos hasta el Neolítico Final (Valera 2009).

(2) El sondeo 1 se llevó a cabo sobre lo que, según las fotografías aéreas, parecía uno de los fosos interiores, concretamente en un tramo del mismo al sur de la plataforma central del lugar. El sondeo 2 se ubicó al noroeste del círculo central, en una zona donde previamente, gracias a las prospecciones superficiales, se había localizado una concentración de artefactos relacionados con la actividad metalúrgica. El sondeo 3 se centró en lo que según las fotografías aéreas del momento parecía ser el foso más interior. El sondeo 4 fue llevado a cabo en la necrópolis, iniciándose la excavación del llamado sepulcro 1. El sondeo 5, 1998b). Estos trabajos de campo precisaron aún más la fisonomía del yacimiento, configurándose una imagen bastante aproximada sobre la que durante más de diez años se articularon los trabajos posteriores (Fig. 3).

No obstante, desde ese momento y hasta 2006 los esfuerzos se concentraron en el sector de la necrópolis, continuándose la excavación del sepulcro 1, comenzada en 1997, e iniciándose la del sepulcro 2. Ambos presentaban una morfología parecida; cámara circular, corredor y atrio (Valera et al. 2000). Se documentó, sin llegar a excavarse, una estructura en negativo con abundantes fragmentos óseos y cerámicos. Por hallarse también en el área de necrópolis, fue considerada de forma preliminar como una posible sepultura colectiva en fosa simple (sepulcro 3) (Valera et al. 2007: 55).

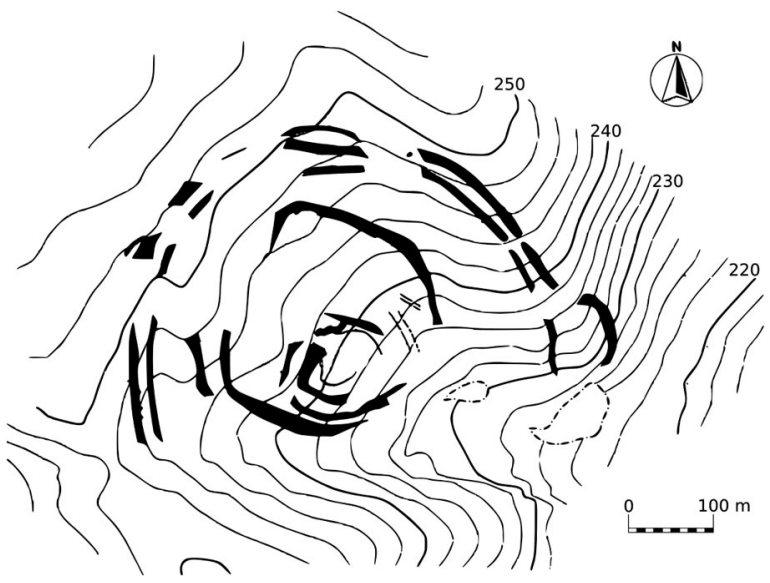

Fig. 3. Perdigões (Reguengos de Monsaraz, Portugal). Planta del yacimiento, reconocida a partir de las fotos aéreas de 1997 (Lago et al. 1998a: fig. 2).

\section{PROGRAMA GLOBAL DE INVESTIGACIÓN ARQUEOLÓGICA DE PERDIGÕES}

En 2006 hubo un cambio en el proceso de investigación del sitio, de modo que desde entonces todas las actuaciones arqueológicas se vienen enmarcando dentro del denominado Programa Global de Investigación Arqueológica de Perdigões (INARP), responsabilidad del Núcleo de Investi-

finalmente, se localizó en el foso más externo, en las cercanías de la denominada puerta noreste. 
gación Arqueológica (NIA) de Era-Arqueología S.A (Valera et al. 2008).

Dicho programa busca establecer las líneas orientativas para una política integrada de la investigación en el lugar. La idea de partida es que el crecimiento y el desarrollo del estudio -tanto a nivel de financiación como de problemáticas científicas-debe potenciarse con la participación de otras instituciones y equipos de investigación, portugueses y extranjeros. Consecuentemente, se han abierto nuevos proyectos o subprogramas de investigación. La tarea de coordinación que garantiza la gestión integrada de las diferentes líneas de investigación, que asegura los niveles básicos de replicabilidad entre los diferentes proyectos y que establece los criterios o prioridades del trabajo se reserva a la propia Era-Arqueología, a través del NIA. Todo lo dicho ha permitido que, a fecha de hoy, ya estén funcionando diversos equipos científicos embarcados en varios proyectos o subprogramas con objetivos más concretos (3) (Valera et al. 2008).

En esta coyuntura, en 2007-2008 Era-Arqueología retomó las excavaciones fuera de la necrópolis, en un sector que abarcaba parte de un foso doble en la zona intermedia del complejo de recintos (Valera 2008a, 2008b). Finalmente en 2009 comenzaron las intervenciones en la zona central del yacimiento, en un área que abarcaba parte de la mancha oscura visible en las fotografías aéreas, entre otros elementos (Valera 2009).

Paralelamente, el Área de Prehistoria de la Universidad de Málaga (UMA) fue invitada a participar desarrollando un proyecto propio que se integraría, como un subprograma, en el marco general arriba descrito. Así, y atendiendo a la línea de investigación abierta en tal universidad desde finales de los años 90 sobre los yacimientos de fosos (Jiménez 2006-2007, 2007; Jiménez y Márquez 2006, 2010; Márquez 2001, 2003, 2004, 2006a, 2006b, 2007; Márquez y Fernández Ruiz 2002; Márquez y Jiménez 2008, 2010a, 2010b; Márquez et al. 1999) se creó el Proyecto

(3) Podemos apuntar: Ambientes funerários (Era-Arqueología; Universidade do Algarve) Actividade Metalúrgica (Era-Arqueología; Instituto Tecnológico y Nuclear; Universidade Nova-CENIMAT); Relação Homen/Animal e ontologia humana no Neolítico e Calcolítico (Era-Arqueología; Igespar; Universidade do Algrave); Produções ceramicas (Instituto Tecnológico y Nuclear); Antropologia das Populações dos Perdigões (Universidade de Coimbra; Era-Arqueología); Produções líticas (Universidade do Algarve). de Investigación "Excavaciones en el yacimiento de Perdigões 2008-2010 (Universidad de Málaga, España)".

Los objetivos generales y específicos de este subprograma han sido ya adelantados (Márquez et al. 2008). La iniciativa pretende, preferentemente y como objetivo marco, participar activamente en las discusiones sobre metodología e interpretación de los recintos de fosos que pudieran surgir al amparo del INARP (Márquez et al. 2008: 42), e incrementar nuestro conocimiento sobre la planta general del recinto (Márquez et al. 2008: 44). En función de los objetivos iniciales, se ha reservado la Puerta NE del recinto exterior (sector L) como zona específica de trabajo para el equipo de la Universidad de Málaga. Allí se busca obtener más datos acerca de la morfología de la entrada, la temporalidad de los fosos exteriores y su posible relación con la necrópolis que se encuentra entre ellos (Márquez et al. 2008: 43). Los primeros trabajos de la UMA se emprendieron en agosto de 2008.

\section{PROSPECCIONES GEOFÍSICAS EN PERDIGÕES. CAMPAÑAS 2008 Y 2009}

\subsection{Georadar}

Las primeras actuaciones del equipo de investigadores de la Universidad de Málaga en el Complexo Arqueológico dos Perdigões consistieron en prospecciones geofísicas previas a la excavación arqueológica. En coordinación con el NIA de Era, durante el mes de agosto de 2008, se proyectaron sondeos mediante la técnica de georadar a cargo de la empresa Eastern Atlas Geophysical Prospection (4).

De acuerdo con los objetivos generales arriba descritos, las labores prospectivas se aplicaron a tres áreas específicas elegidas de forma consensuada (Fig. 4): a) Sector Puerta NE, es decir, la zona específica de actuación de la UMA, donde

(4) Las prospecciones fueron financiadas con recursos provenientes del Proyecto de Investigación I+D+i HUM200763419/HIST de la Secretaría de Estado de Universidades e Investigación del Ministerio de Ciencia e Innovación, desarrollado en la Universidad de Málaga, y titulado: "Repensando Tartesos bajo el prisma de la identidad: el componente fenicio". Por su parte, las labores de campo fueron asumidas por un equipo formado conjuntamente por miembros de la UMA y de Era. 


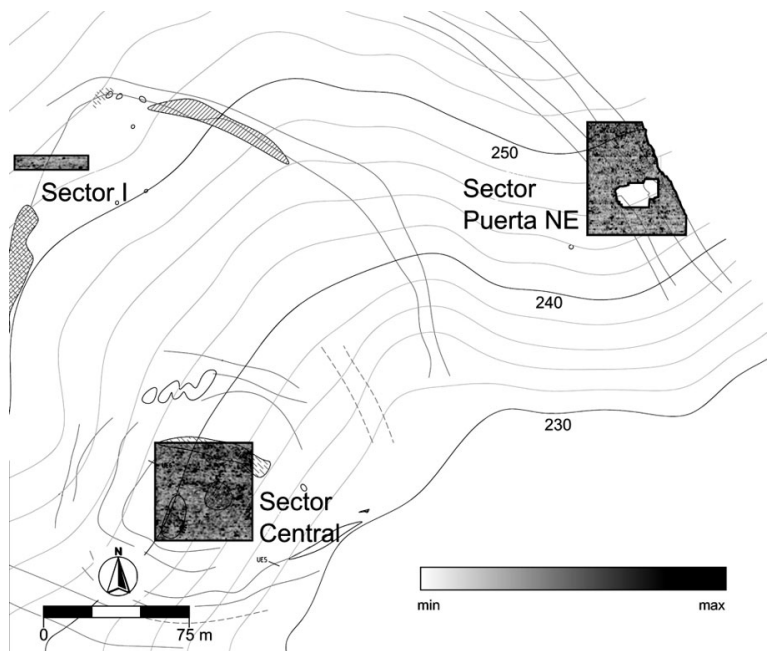

Fig. 4. Perdigões (Reguengos de Monsaraz, Portugal). Resultado de la prospección con georadar a cota 0,80-1,20 m (agosto 2008). Tiempo transitorio: $14 \mathrm{~ns}-21$ ns. Resolución 0,5 m × 0,02 m. Imagen 0,25 m ×0,25 m. Equipo SIR - 3000, antena de $270 \mathrm{MHz}$.

se efectuaron mediciones a lo largo de $1500 \mathrm{~m}^{2}$; b) Sector Central, de $1620 \mathrm{~m}^{2}$, coincidiendo con la gran mancha circular que aparecía en las fotografías aéreas de 1997; y c) Sector I, de sólo 195 $\mathrm{m}^{2}$, en el área intermedia, tangencialmente a las excavaciones que en aquellos mismos momentos (julio-agosto de 2008) realizaba Era en uno de los fosos interiores (Valera 2008a, 2008b). Este último sector se eligió con la intención de evaluar las prestaciones del método en una zona que simultáneamente se empezaba a conocer por los sondeos arqueológicos.

Los resultados de las prospecciones con georadar fueron muy desiguales y el método resultó poco adecuado. Así, si bien en el primer sector (Puerta NE) se confirmaba la existencia de una discontinuidad que, con toda probabilidad, debía de ser la puerta buscada, la información no mejoraba la de las fotos aéreas de 1997. Por su parte, la mancha central, según el georadar, no se relacionaba con estructuras negativas, sino con una dispersión de bloques de piedra y materiales arqueológicos, pero seguía quedando muy mal definida. Por último, en el Sector I los resultados fueron contradictorios con la excavación arqueológica que se estaba realizando en sus inmediaciones, al no documentar las prospecciones geofísicas los fosos y fosas que aquélla mostraba fidedignamente.
En general, se advirtió que el georadar no era un método efectivo en las condiciones existentes. Las causas se podían encontrar tanto en la falta de contrastes geofísicos entre las estructuras arqueológicas y el suelo natural, como en la realización de las mediciones en sectores quizá demasiado limitados en extensión para proporcionar resultados significativos. En cualquier caso, la coyuntura aconsejaba cambiar de estrategia y probar con otro tipo de prospecciones y con áreas de trabajo de mayor superficie.

\subsection{Prospecciones geomagnéticas}

Éstas se llevaron a cabo en junio y septiembre de 2009 (5), quedando a cargo de la empresa Becker Archaeological Prospection. En la campaña de junio, como había ocurrido en 2008 con el georadar, los trabajos de magnetometría se limitaron a evaluar, a modo de "test", la idoneidad del método en relación con las características del sitio. Se decidió prospectar un área que, de dentro a fuera, uniera el espacio central (la gran mancha) con la Puerta NE. Es decir, se volvía sobre áreas sondeadas el verano anterior con georadar, extendiendo las mediciones a los terrenos intermedios que quedaban entre uno y otro sector.

En los sondeos geomagnéticos se empleó el magnetómetro de cesio Geometrics $G-858 G$, en configuración móvil de doble sensor, con una separación entre sensores de $0,5 \mathrm{~m}$. Este método para medir el campo geomagnético total logra la mayor sensibilidad (20 picotesla), penetración y velocidad (20 muestras por segundo) para cubrir grandes áreas (más de 1 ha por día, con una resolución de $10 \times 50 \mathrm{~cm}$ ). La superficie total prospectada en Perdigões, en 16 días, asciende a casi 20 ha (122 cuadrículas de $40 \times 40 \mathrm{~m}$ ), acumulándose un total de 3,9 millones de medidas.

Los resultados fueron tan satisfactorios que se decidió, de acuerdo con los objetivos del INARP

(5) Las prospecciones geomagnéticas desarrolladas tanto en junio como en septiembre de 2009 fueron sufragadas por la Consejería de Innovación, Ciencia y Empresa de la Junta de Andalucía, a través del Proyecto de Investigación de Excelencia P08-HUM-04212 "Estudio Arqueológico y Gestión Patrimonial en los recintos de fosos del suroeste de la Península Ibérica (Andalucía, Algarve, Alentejo)". Los trabajos de campo fueron llevados a término y coordinados por un equipo de la UMA y otro de Era-Arqueología. Esta última institución fue especialmente responsable del planeamiento de cuadrículas previo a los sondeos, además de correr con determinados gastos de logística. 
y de los del proyecto de la UMA, prospectar toda el área comprendida entre los dos fosos exteriores. Por cuestiones de logística, la continuación de las labores de magnetometría se pospuso hasta septiembre. Además, los resultados de esta primera fase fueron tan ilustrativos que orientaron, en gran medida, las excavaciones arqueológicas que, durante julio-agosto de 2009 realizaron tanto Era en la zona central como la UMA en la Puerta NE.

Durante una quincena de septiembre se completaron los análisis geomagnéticos en todo el área de trabajo. Sólo se dejaron de prospectar: a) el carril o pista que atraviesa de $\mathrm{E}$ a $\mathrm{O}$ el yacimiento en su parte meridional; b) una amplia zona al E del mismo, donde una viña plantada impedía la aplicación del método y c) una estrecha sección al sur del conjunto en la que, por las tareas agrícolas en ella desempeñadas, también resultaba inviable la toma de datos. Pese a estos déficits de información, la composición de los resultados de las mediciones geomagnéticas en una imagen supone todo un éxito en cuanto a la calidad y nitidez de la misma (Fig. 5).

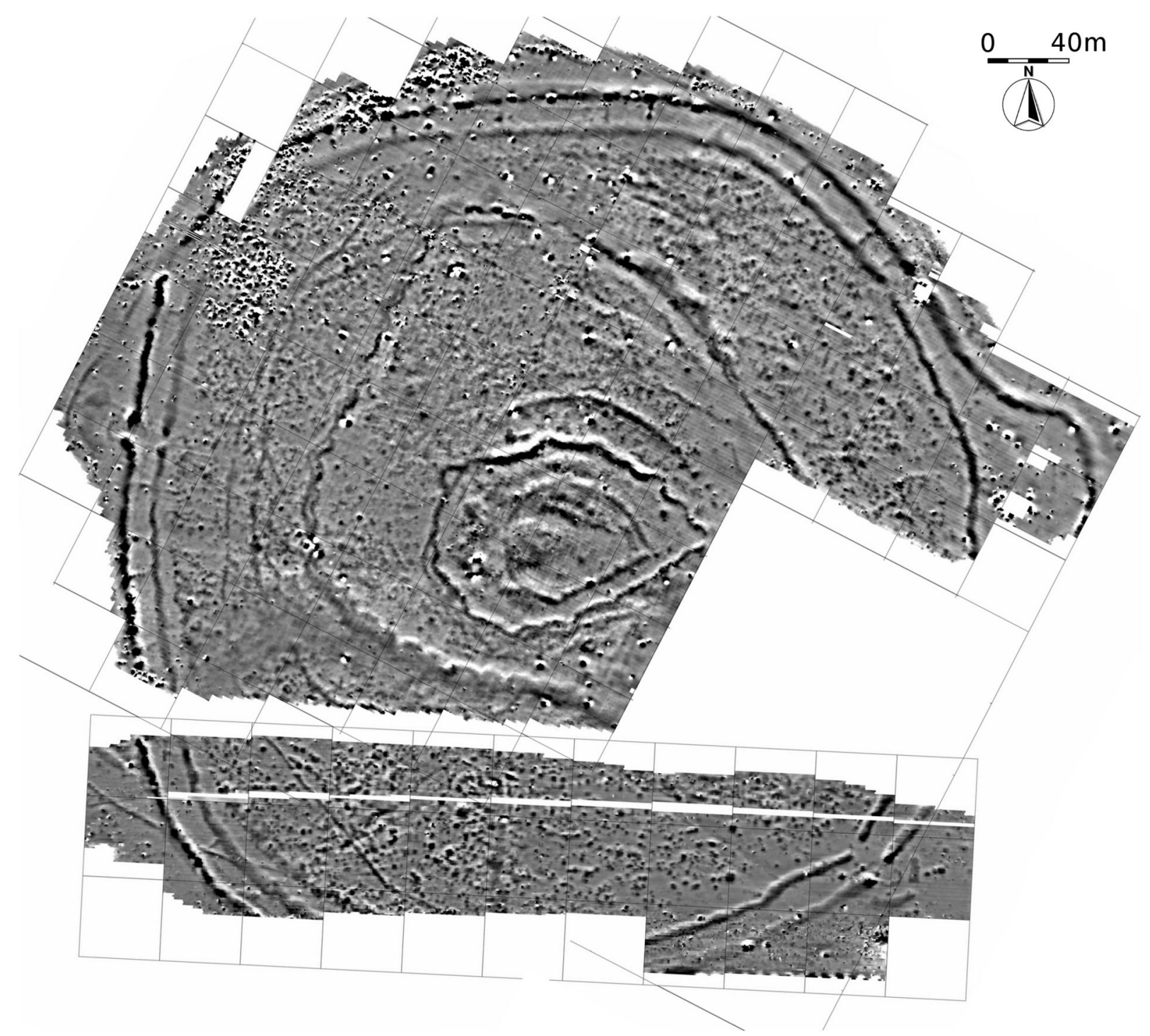

Fig. 5. Perdigões (Reguengos de Monsaraz, Portugal). Planta de los recintos de fosos de Perdigões, de acuerdo con la magnetometría realizada en el verano de 2009. 


\section{PROSPECCIONES GEOMAGNÉTICAS EN PERDIGÕES. UNA PRIMERA LECTURA}

Procede, a continuación, realizar una lectura inicial de los distintos elementos arquitectónicos que formaron parte estructural del yacimiento, según se desprende de las prospecciones geomagnéticas de 2009. Conviene tener en cuenta que las últimas excavaciones, desempeñadas por Era (2007-2009) y la UMA (2009-2010), vienen poniendo de manifiesto la prolongada temporalidad del yacimiento, que pudo ser de más de 1500 años, con anillos que se construyeron cuando otros más antiguos ya se habían colmatado (Valera 2009). No obstante, una lectura precisa por fases requeriría un apoyo documental mucho mayor del que podemos exponer en este instante, debiendo quedar, por tanto, aplazada para momentos más avanzados de la investigación. En consecuencia, la descripción que sigue sólo deta1la las características generales del conjunto tal y como ha llegado a nosotros, sin considerar la cronología de cada una de las estructuras.

Lo primero que salta a la vista es que en Perdigões se distribuyen, cuando no se solapan, hasta 11 o más anillos de tendencia concéntrica, constituidos, bien por zanjas o fosos excavados en la roca basal, bien por posibles empalizadas. En segundo lugar, no es menos elocuente la profusa presencia de centenares de estructuras tipo hoyo o fosa que se distribuyen entre los diversos recintos.

Para facilitar la descripción, discriminaremos tres zonas específicas. Esta parcelación no debe ser entendida en términos arquitectónicos, funcionales o históricos, sino tan sólo como recurso expositivo. Nos ayudaremos de un calco preliminar realizado sobre la imagen en bruto, que no debe verse en modo alguno como una lectura final o definitiva de la planimetría (Fig. 6).

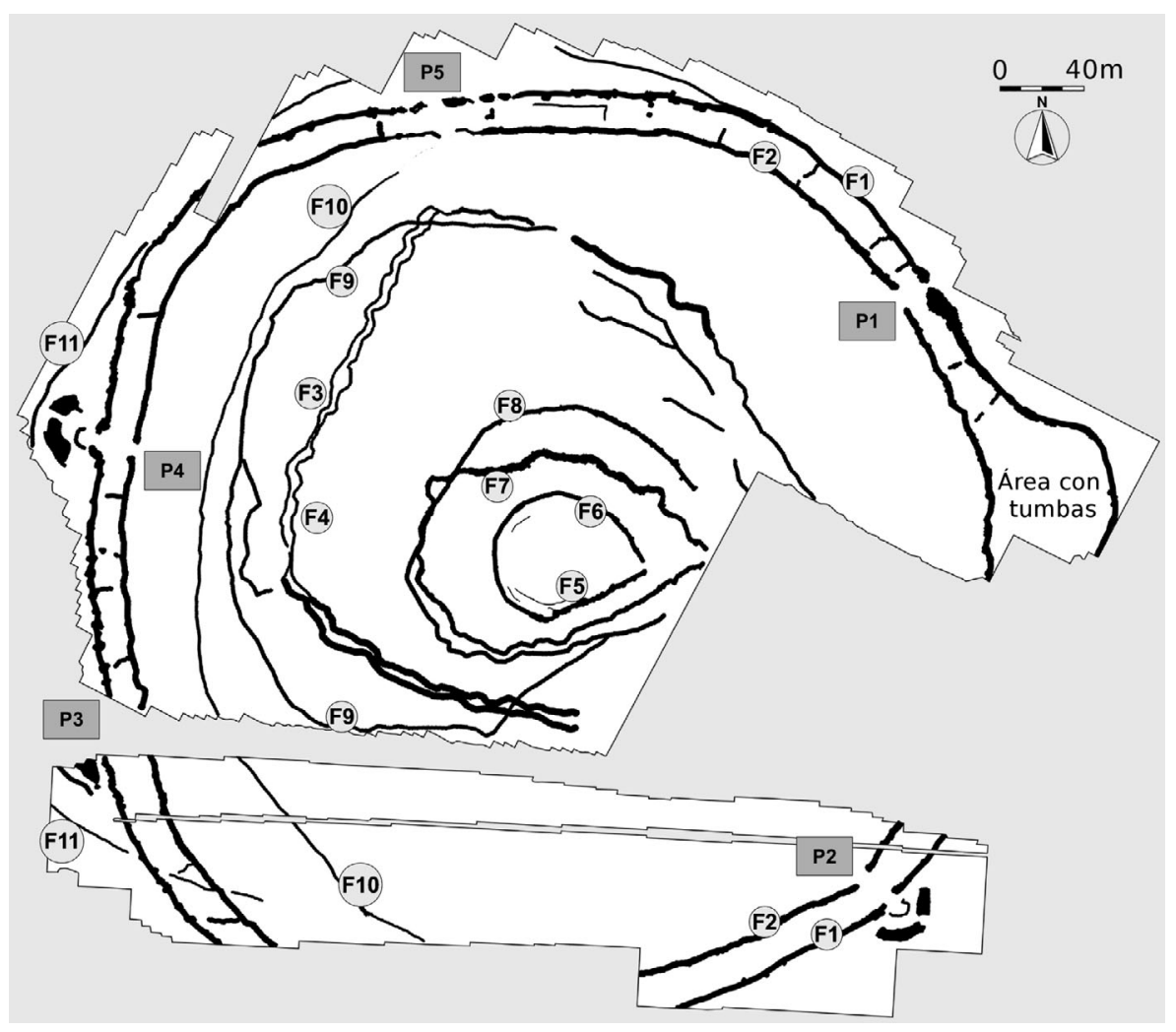

Fig. 6. Perdigões (Reguengos de Monsaraz, Portugal). Ensayo de calco y primera lectura - por tanto, provisional- de las realidades arqueológicas desveladas a través de la prospección geomagnética: 11 fosos $(\mathrm{F})$ y 5 puertas $(\mathrm{P})$. 


\section{1. Área central}

Esta zona interior del conjunto se ve menos afectada por los espacios no prospectables. En ella podemos percibir, al menos, tres claros recintos concéntricos (F6, F7, F8) (6). De dentro a fuera, se observa que los dos primeros muestran tendencia ojival y unas posibles puertas en sus extremos convergentes (orientadas al E). El más interior (F6) parece albergar, además, una (¿doble?) empalizada paralela (F5), bien identificable en su recorrido más meridional. El segundo (F7) presenta un perfil sinuoso. Por último, el tercero de los fosos centrales (F8) es el peor definido. No queda claro si participa o no de la tendencia ojival de los anteriores. Su planta podría ser subcircular (aunque un segmento al S sugiere una planta semejante a la de los anteriores), compartiendo con ellos una distribución concéntrica. Este F8 aparece muy difuso en su recorrido, especialmente en el cuadrante sur-sureste. Es más, en numerosos momentos parece solaparse y entrecruzarse con el trazado de F7, sin que por el momento tengamos clara la relación de anterioridad o posterioridad entre uno y otro.

En el espacio donde en las fotografías aéreas de 1997 podíamos ver una mancha negruzca, (Fig. 2) se observa una anomalía magnética, bien destacada respecto de su entorno y de color gris oscuro, que groseramente se corresponde con aquélla, ocupando el centro geométrico del yacimiento, y afectando, en planta, de forma tangencial, al primero de los fosos y a su posible empalizada interior (F5 y F6) (7).

La superficie interior a estos primeros recintos es, comparativamente, la que parece concentrar un número menor de estructuras tipo hoyo, lo que no quita que se aprecien, en cualquier caso, un número considerable de ellas.

(6) Se sigue la numeración existente en el registro, condicionada por el orden y el método de identificación de los distintos anillos (fotografía aérea, prospecciones geofísicas).

(7) Las excavaciones de Era en 2009 han permitido, en primera instancia, identificar en ese espacio de color oscuro una estructura negativa y otra positiva. La negativa podría constituir otro foso, cuyos materiales de relleno apuntan a un Calcolítico Pleno local, mientras el cuerpo en positivo se corresponde con una gran estructura de piedra, todavía sin definir claramente. Aparentemente es de época campaniforme, es decir, posterior, tanto al hipotético foso que la acompaña como, más aún, a los anillos F5 y F6, a los que corta (Valera 2010).

\section{2. Área o cinturón intermedio}

Consta de, al menos, otros tres o cuatro recintos de mayores dimensiones (F3, F4, F9, y F10). De dentro a fuera, F3 y F4 conforman un recinto doble, o dos recintos paralelos, en casi la totalidad de su trazado perceptible en la imagen (Valera 2008a, 2008b). Presentan una tendencia no circular, si bien la inexistencia de información en la parte oriental impide, por el momento, una lectura más segura de su configuración. Su trazado, como ocurría con F7, es manifiestamente sinuoso en la mayoría de su recorrido.

Los anillos F9 y F10 son especialmente difíciles de interpretar pese a que ninguna de sus plantas contradice la naturaleza concéntrica del conjunto. En primer lugar, porque ambos, y especialmente el F9, se hallan muy afectados por los vacíos de la prospección. En segundo lugar, porque el recinto doble y sinuoso (F3 y F4) se solapa con F9. Lo que sí está claro, a tenor del mapa geomagnético, es que estos fosos son aparentemente más estrechos que el resto de los documentados.

Los tres recintos descritos no agotan las estructuras observables en el área intermedia. Hay una importante concentración de posibles fosas circulares y cubetas que se distribuyen en el área comprendida entre estos nuevos recintos y los que describimos en el área interior (8). Se aprecian también tramos parciales o incompletos de otros fosos o empalizadas mal definidos y cuya identificación, casi intuitiva, dificulta y complica innecesariamente la descripción, por lo que los obviamos en este momento.

\section{3. Área 0 cinturón exterior}

Sin lugar a dudas, en esta zona externa es donde mejor se perciben los elementos arquitectónicos del yacimiento. Básicamente se trata de dos anillos (F1 y F2) constituidos por dos anchos fosos. Presentan un trazado paralelo y, en su desarrollo, se integran de forma pareada hasta 5 accesos. En la zona oriental, como se conocía desde 1997, la zanja más externa (F1) modifica la tendencia subcircular de su recorrido, separándose de su foso adyacente (F2), hasta configurar el espacio donde se ubican las estructuras funerarias

(8) Las excavaciones de 2007-2008 han puesto de manifiesto la presencia de restos osteológicos humanos (Valera 2008b; Godinho 2008). 
de tipo tholos. La armonía advertida entre ambos fosos, que podría suponer también su contemporaneidad, se reafirma, más si cabe, mediante una serie de zanjas o tramos interiores de empalizadas que, perpendicularmente, a modo de "tirantes", parecen unirlos a intervalos más o menos regulares. Hasta 16 ó 17 posibles "tirantes" se hacen visibles a partir de la magnetometría.

Adicionalmente, al interior de la antepenúltima zanja (F2) se deja ver, en toda su extensión, una franja de terreno vacía, es decir, notablemente libre de cualquier estructura de tipo hoyo, que tanto abundan en el resto de la superficie estudiada. Este espacio exento, en yacimientos europeos de naturaleza comparable, se suele identificar con el área originariamente ocupada por un bank o terraplén, hipotéticamente construido con los materiales extraídos al romper la roca virgen en el momento de excavar las estructuras, constituyendo un anillo gemelo al foso adyacente, pero en positivo. En Perdigões, este comportamiento, al menos de forma tan patente, no se ha podido reconocer en el resto de los anillos ya descritos.

Quizá una de las mayores novedades aportadas por las prospecciones geomagnéticas de 2009 es la documentación de hasta 5 puertas en el cinturón exterior del yacimiento. Siguiendo la dirección de las agujas del reloj, a las dos puertas conocidas hasta el momento, la NE (P1) y la SE (P2), se añaden tres posibles nuevas entradas: la $\mathrm{P} 3$, orientada al $\mathrm{O}-\mathrm{SO}$, deducida más por la fisonomía del espacio en cuestión que por su constatación real; la P4, apuntando hacia el O; y la P5, alineada grosso modo con el Norte. En todos los casos las aperturas atraviesan perpendicularmente los dos fosos delimitadores del conjunto (F1 y F2). Las puertas P2 (SE) y P4 (O), y quizás la P3 (O-SO), ofrecen suficiente información para ver en ellas accesos complejos o monumentales. Nos detendremos brevemente en este punto.

La P2 se abre al SE. Se tenía conocimiento de la misma por la toma de las fotografías aéreas de 1997 y por imágenes posteriores obtenidas a través de Google Earth. La magnetometría ha permitido describir con mayor precisión su naturaleza arquitectónica, aunque de ninguna manera sería prudente descartar el carácter acumulativo, y por tanto diacrónico, de sus elementos. En el tramo que describe la puerta a la altura del F2 no se aprecia recurso alguno más allá de la propia discontinuidad del mismo. En cambio, la abertura del F1 presenta, exteriormente, algunos elemen- tos desconocidos hasta el momento en el sitio. En primer lugar, una zanja (o quizá cimiento de estructura de madera) semicircular que, a modo de "ímbrice", interrumpe o dificulta el acceso. Recuerda a las frecuentes estructuras llamadas de tipo fence que abundan en los recintos europeos de la misma época. En segundo lugar, dos tramos de zanja de gran anchura, de tendencia semicircular, aunque con una discontinuidad intermedia. Estas últimas construcciones añaden al conjunto un recurso semejante a las célebres "pinzas de cangrejo", tan típicas, aunque no exclusivas, del Neolítico en el territorio francés (Márquez y Jiménez 2010a). Ahora bien, al no arrancar, aparentemente, desde el foso exterior, resultaría un tipo formalmente distinto a los modelos clásicos.

La P4, hasta el momento desconocida, presenta en el extremo de ambos fosos y donde se abre la propia discontinuidad, un ligero exvasado de sus jambas. Por lo demás, su forma es semejante a la P2. Al exterior del F1 vuelve aparecer el elemento que, como "ímbrice" o fence, complica el acceso y, con menor claridad, los tramos de zanja que, en forma de arco, evocan las "pinzas de cangrejo". En definitiva, la trama de la P4 nos lleva a pensar que el peculiar diseño de la Puerta 2 proporcionado por la prospección geomagnética no es casual ni anómalo. Al menos con los resultados de la magnetometría en la mano, se podría proponer un modelo de acceso característico de Perdigões, que podríamos llamar "puerta de ímbrice" o, simplemente, "entrada tipo Perdigões". Este tipo de solución, a pesar de la ausencia de información geomagnética debido a las interferencias metálicas junto a los caminos, no es descartable en otras entradas. Todo ello, repetimos, sin menoscabo de que la planta del sitio en esos dos sectores pueda ser en realidad el sumatorio de actuaciones constructivas llevadas a cabo en sucesión diacrónica, lo que sólo podrá ser aclarado por excavaciones arqueológicas ejecutadas al efecto.

Cabe apuntar que, al exterior de la zanja F1, a la altura de las Puertas 4 y 5, se aprecia el trazado de un foso (F11). Por el momento constituye la línea más externa conocida, pero podría prolongarse por el sur hasta cruzarse con algunos de los recintos interiores, si bien la planta del yacimiento es tan complicada y los datos de esta zona son tan escasos que ninguna afirmación al respecto puede ser rotunda. 
Sí resulta muy significativo que la superficie comprendida entre el foso F3, en la zona intermedia, y el F2, ya en el cinturón exterior, es la parcela donde de forma más notoria se acumulan centenares de estructuras circulares que reconocemos como hoyos o fosas. Algunas fueron excavadas por Era en el verano de 2008 (Valera 2008a, 2008b) ya reproducían en su forma y relleno las que, por millares, se documentan en otros yacimientos de la Península Ibérica y de otras regiones europeas.

\section{CONSIDERACIONES FINALES}

Tras ser descubierto en 1983, Perdigões se ha convertido, a fecha de hoy, en uno de los yacimientos imprescindibles para conocer la Prehistoria reciente de la Península Ibérica. En este trabajo hemos repasado las principales etapas de la investigación en el lugar. Además, hemos subrayado la filosofía de colaboración entre equipos e instituciones que surge al amparo del INARP, recientemente publicada (Valera et al. 2008). Por último, hemos dado a conocer de forma novedosa y preliminar las prospecciones geofísicas llevadas a cabo durante 2008 y 2009 por parte de la Universidad de Málaga, con la colaboración de Era-Arqueología.

Los resultados obtenidos con el método geomagnético corroboran muchos de los pormenores reconocidos desde 1997, cuando se hicieron las primeras tomas aéreas del lugar. Tal es el caso de los dos grandes fosos que circunscriben el sitio, con el manifiesto embolsamiento que exhibe el más exterior de ellos. Asimismo, la geomagnética muestra nítidamente el trayecto de algunas zanjas, como las del área intermedia, que apenas se vislumbraban en la foto aérea.

Pero, sobre todo, la prospección geomagnética ha propiciado la detección de elementos absolutamente novedosos en el yacimiento. Nos referimos, por ejemplo, a la identificación de nuevos anillos de fosos, algunos sinuosos, y tramos de aparentes empalizadas. Pensamos también en la multiplicación exponencial de los datos referentes a las entradas a los distintos recintos, destacando sobremanera la proliferación de puertas monumentalizadas en el foso $\mathrm{F} 1$, que podrían servir para definir un nuevo tipo formal en el contexto europeo: la "puerta de ímbrice" o de tipo Perdigões. Por último, no deja de sorpren- der la exuberante proliferación de estructuras circulares tipo fosa u hoyo, que en ingentes cantidades se disponen, frecuentemente solapadas, en el interior de los diversos espacios.

En suma, la "radiografía" de conjunto con la que contamos en estos momentos del complejo de Perdigões presenta una definición extraordinaria que nos posibilita apreciar la especial complejidad estructural de los recintos de fosos peninsulares. Por lo que sabemos hasta estos instantes, la imagen es acumulativa, y no se puede interpretar en términos históricos absolutos. Sin embargo permite y favorece una planificación bien fundada de los futuros trabajos arqueológicos, cuyos objetivos paralelos estarán orientados a solucionar los problemas de temporalidad de los múltiples elementos arquitectónicos identificados.

En última instancia, creemos que la utilización combinada de técnicas como la fotografía aérea, las prospecciones geofísicas y las excavaciones arqueológicas es el método recomendable para estudiar estos yacimientos. Incidir en sondeos arqueológicos descontextualizados o análisis puntuales de las estructuras y sus rellenos nos condenan a una perspectiva muy limitada del fenómeno arqueológico de los recintos de fosos.

\section{AGRADECIMIENTOS}

La mayor parte de los terrenos prospectados pertenecen a la empresa Esporão S.A. La amabilidad y colaboración continuada de su propietario, José Roquette, facilitaron las actuaciones arqueológicas. Por igual motivo, agradecemos la cooperación de los restantes propietarios: Rolando Moniz Simões Palma, Fernando Manuel Ramos Marcão y Gertrudes Caeiro Gonçalves.

Germán Delibes y José A. Rodríguez, así como Jesús Sesma y Jesús García, nos facilitaron el acceso directo a los resultados inéditos provenientes de las prospecciones geomagnéticas en El Casetón de la Era, en Valladolid, y Los Cascajos, en Navarra, respectivamente. Contamos además con sus comentarios y sugerencias sobre las posibilidades del método en yacimientos de fosos como el de Perdigões. Por último, en la preparación y el transcurso de los trabajos fue central la colaboración y logística aportada por Miguel Lago, de Era-Arqueología S.A. 


\section{BIBLIOGRAFÍA}

Godinho, R. 2008: "Deposições funerárias em fossa nos Perdigões: dados antropológicos dos Sector I". Apontamentos de Arqueologia e Património 3. NIA-ERA Lisboa: 29-34.

www.nia-era.org/content/view/280/33 (consulta 8-IV-2011).

Gomes, M. V. 1994: "Menires e cromeleques no complexo cultural megalítico português - trabalhos recentes e estado da questão". Actas do seminário ' $O$ Megalitismo no Centro de Portugal: novos dados, problemática e relações com outras áreas peninsulares' (Mangualde, 1992): 317-342. Viseu.

Gonçalves, V. S. 1999: Reguengos de Monsaraz: Territórios megalíticos. Câmara Municipal de Reguengos de Monsaraz. Lisboa.

Jiménez, V. 2006-2007: "Pithouses versus pits. Apuntes para la resolución de un problema arqueológico". Portugalia nova série XXVII-XXVIII: 35-48.

Jiménez, V. 2007: "La Premisa Pompeya y las 'cabañas semisubterráneas' del sur de la Península Ibérica (IV-III milenios AC)". Mainake XXIX: 475-492.

Jiménez, V. y Márquez, J. E. 2006: “AAquí no hay quien viva'. Sobre la existencia de casas-pozo en Andalucía durante el IV y III milenios AC". Spal, Revista de Prehistoria y Arqueología 15: 39-49.

Jiménez, V. y Márquez, J. E. 2010: "Structured deposition and ditched enclosures in the Late Prehistory of Southern Iberia (IV-III millennia BC)". Proceedings of the XVth World Congress of the International Union for Prehistoric and Protohistoric Sciences (IUPPS) (Lisboa, 2006) 7. British Archaeological Reports, International Series 2122, Archaeopress. Oxford: 145-152.

Lago, M.; Duarte, C.; Valera, A.; Albergaria, J.; Almeida, F. y Carvalho, A. 1998a: "Povoado dos Perdigões (Reguengos de Mosaraz): dados preliminares dos trabalhos arqueológicos realizados em 1997'. Revista Portuguesa de Arqueologia 1 (1): 45-152.

Lago, M.; Duarte, C.; Valera, A.; Albergaria, J.; Almeida, F.; Carvalho, A. y Reis, S. 1998b: Povoado Pré-Histórico dos Perdigões (Reguengos de Mosaraz). Relatório final dos trabalhos de salvamento arqueológico. Relatórios Era 1. Era-Arqueología.

Leisner, G. y Leisner, V. 1951: Antas do concelho de Reguengos de Monsaraz. UNIARCH. Lisboa.

Márquez, J. E. 2001: “De los campos de silos a los agujeros negros': sobre fosas, depósitos y zanjas en la Prehistoria Reciente del Sur de la Península Ibérica”. Spal, Revista de Prehistoria y Arqueología 10: 207-220.

Márquez, J. E. 2003: "Recintos Prehistóricos atrincherados (RPA) en Andalucía (España): Una propuesta interpretativa". En S. O. Jorge (ed.): Recintos murados da Pré-história recente. Universidade do Porto. Porto: 269-284.

Márquez, J. E. 2004: "Muerte ubícua: sobre deposiciones de esqueletos humanos en zanjas y pozos en la prehistoria reciente de Andalucía". Mainake XXVI: 115-138.

Márquez, J. E. 2006a: "Sobre los depósitos estructurados de animales en yacimientos con fosos del sur de la Península Ibérica”. En E. Weiss-Krejci (ed.): Animais na Pré-história e Arqueología da Península Ibérica. Actas do IV Congresso de Arqueología Peninsular (Faro, 2004): 15-25. Faro.

Márquez, J. E. 2006b: "Neolithic and Copper Age ditched enclosures and social inequality in the Iberian south (IV-III millennia cal BC)"'. En P. Díaz-delRío y L. García Sanjuán (eds.): Social Inequality in Iberian Late Prehistory, British Archaeological Reports International Series 1525. Hadrian Books/Basingstoke Press/. Archaeopress. Oxford: 171-187.

Márquez, J. E. 2007: "La problemática de los recintos de fosos de la Prehistoria Reciente en el sur de España”. En S. O. Jorge, A. M. Bettencourt e I. Figueiral (eds.): "A concepção das paisagens e dos espaços na Arqueologia da Península Ibérica". Actas do IV Congresso de Arqueologia Peninsular (Faro, 2004): 27-35. Faro.

Márquez, J. E. y Fernández, J. 2002: "Viejos depósitos, nuevas interpretaciones: La estructura n. ${ }^{\circ} 2$ del yacimiento prehistórico de los Villares de Algane (Coín, Málaga)". Mainake XXI-XXII: 301-333.

Márquez, J. E.; Fernández, J. y García, M. 1999: “Un asentamiento prehistórico en el casco urbano de Alameda (Málaga)". Baetica 21: 177-206.

Márquez, J. E. y Jiménez, V. 2008: “Claves para el estudio de los Recintos de Fosos del sur de la Península Ibérica". Era-Arqueología 8: 138-171.

Márquez, J. E. y Jiménez, V. 2010a: Recintos de Fosos. Genealogía y significado de una tradición en la Prehistoria del suroeste de la Península Ibérica (IV-III milenios $A C$ ). Servicio de publicaciones de la Universidad de Málaga. Málaga.

Márquez, J. E. y Jiménez, V. 2010b: "Ten keys to think southern Iberian ditched enclosures". Proceedings of the XVth World Congress of the International Union for Prehistoric and Protohistoric Sciences (IUPPS) (Lisboa, 2006) 36. British Archaeological Reports, International Series 2124, Archaeopress. Oxford: 143-149.

Márquez, J. E.; Jiménez, V. y Mata, E. 2008: “Excavaciones en el yacimiento de Perdigões (Reguengos de Monsaraz, 2080-2010). Universidad de Málaga (España)". Apontamentos de Arqueologia e Património 2. NIA-ERA. Lisboa: 27-34. www.nia-era.org/view/280/33 (consulta 8-IV-2011).

T. P., 68, N. ${ }^{\circ}$ 1, enero-junio 2011, pp. 175-186, ISSN: 0082-5638 doi: $10.3989 /$ tp.2011.11065 
Valera, A. C. 2003: “A propósito dos recintos murados do $4 .^{\circ}$ e $3 .^{\circ}$ milênios a.C: dinâmica e fixação do discurso arqueológico". En S. O. Jorge (ed.): Recintos murados da Pré-história recente. Universidade do Porto. Oporto: 149-168.

Valera, A. C. 2008a: "Intervenção arqueológica de 2007 no interior do recinto Pré-Histórico dos Perdigões (Reguengos de Monsaraz)". Apontamentos de Arqueologia e Património 1. NIA-ERA. Lisboa: 15-22. www.nia-era.org/content/view/280/33 (consulta 8-IV-2011).

Valera, A. C. 2008b: "O recinto calcolítico dos Perdigões: fossos e fossas do Sector I". Apontamentos de Arqueologia e Património 3. NIA-ERA. Lisboa: 19-27. www.nia-era.org/content/view/280/33 (consulta 8-IV-2011).

Valera, A. C. 2008c: "Mapeando o cosmos. Uma abordagem cognitiva aos recintos da Pré-história Recente". Era-Arqueología 8. Era Arqueología/Colibri. Lisboa: 112-127.

Valera, A. C. 2010: "Construção da temporalidade nos Perdigões: contextos neolíticos na área central". Apontamentos de Arqueologia e Património 5. NIA-ERA. Lisboa: 19-26. www.nia-era.org/content/view/280/33 (consulta 8-IV-2011).

Valera, A. C.; Jorge, P. y Lago, M. 2008: “O Complexo Arqueológico dos Perdigões. Breve percurso de uma Arqueologia de minimização a uma Arqueologia em construção e em Sociedade". Almadan II, Série 16: 115-123.

Valera, A. C.; Lago, M.; Duarte, C. y Evangelista L. S. 2000: "Ambientes funerários no complexo arqueológico dos Perdigões: uma análise preliminar no contexto das práticas funerárias calcolíticas no Alentejo". Era-Arqueología 2. Era Arqueologia/Colibri. Lisboa: 84-105. www.nia-era.org/content/view/280/33 (consulta 8-IV-2011).

Valera, A. C.; Lago, M.; Duarte, C.; Dias, I. y Prudêncio, I. 2007: 'Investigação no Complexo Arqueológico dos Perdigões: ponto da situação de dados e problemas". En S. O. Jorge, A. M. Bettencourt e I. Figueiral (eds.): A concepção das paisagens e dos espaços na Arqueologia da Península Ibérica. Actas do IV Congresso de Arqueologia Peninsular (Faro, 2004): 53-66. Faro. 